\title{
Modeling and simulating analysis of bi-motor servo driven system of a large radar
}

\author{
ZHOU Lujun ${ }^{1, a}$, ZHOU Daoqing ${ }^{2, b}$, NIE Yongming ${ }^{3, c}$, RU Hailong ${ }^{4, d}$, HAO \\ Yong ${ }^{5, \mathrm{e}}$ \\ China Satellite Maritime Tracking and Controlling Department, Jiangyin, 214431, China \\ aemail:zhoulujun168@163.com, bemail:623943019@qq.com, email:yimonie@163.com
}

Keywords:Transfer function; Speed loop; Current loop; Potter figure; Transient response

Abstract.Firstly, the control object and the loop of large radar bi-motor servo drive systemis analyzed in detail and the model is given. Then, using the Matlab/simulink simulation software, the system is simulated. At last, the Potter curves and transient response curves of the servo drive system in different states and parameters are obtained, which can provide powerful basis for the analyzing and researching on the system status, parameter changes caused by the servo system performance changing.

\section{Introduction}

In the servo system of large precision tracking radar, usually the typical control methods are adopted, from the inside to the outside are the current loop, speed loop, position loop. Servo drive system generally refers to the part within the speed loop, it drives antenna real-timely and accurately, and ensures the position loop to track the target accurately. After the design of servo system, in the actual application, for environmental factors, equipment modification and performance of components, the system parameters and the load characteristics inevitably will change accordingly[1,2]. In this paper, the author firstly makes models and analyzes of control object and the loop of bi-motor servo drive system of large radar, and use Matlab/simulink simulation software to simulate the system, get the Potter curves and transient response curve of the servo drive system in different states and parameters[3,4]. It provides the powerful basis for the analysis and research on the system status, parameter changes caused by the servo system performance change.

\section{Modeling and analysis of bi-motor servo drive system}

In the bi-motor servo drive system, the current loop of two motor with an antenna axis shares one speed loop, and has only a speed regulator. Speed feedback signal is taken out from the sum of output of the two tachometers, compared with the instruction of the input, through the parallel PID regulator and the acceleration limits, it forms current command and is sent to the current loop of the motor controller. Through a loop regulation and a rectification amplify, it is used to drive the corresponding motor[5-7].

\section{Modeling and analysis of control object}

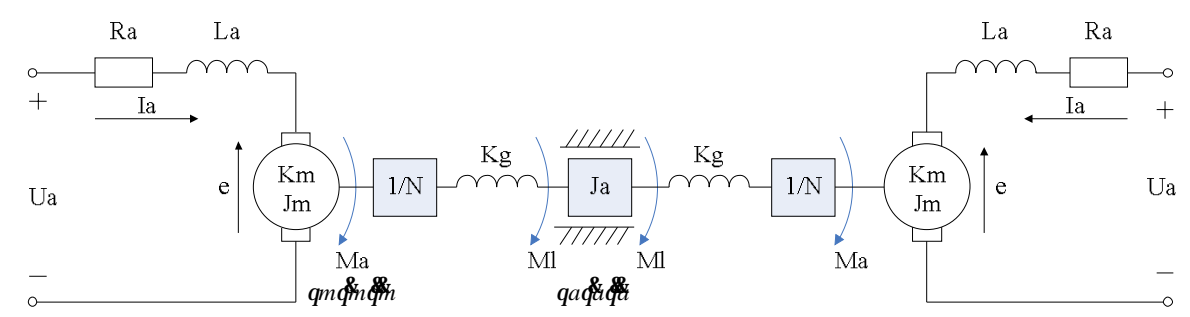

Fig. 1 The model of bi-motor drive

In the figure $1, \mathrm{Ua}$ 、 Ia 、 $\mathrm{Ra} 、 \mathrm{La}$ respectively are motor armature voltage, armature current, armature circuit resistance, total inductance of armature circuit;

$\mathrm{Jm}, \mathrm{Ma}, \mathrm{Km}$ respectively are motor moment of inertia, electromagnetic torque, torque coefficient; 
$\mathrm{E}, \mathrm{Ke}$ respectively are motor back EMF and back EMF coefficient; Ja is antenna inertia;

$\mathrm{N}$ is gear box ratio; $\mathrm{Kg}$ is equivalent stiffness of single gear box output shaft; $\mathrm{Ml}$ is antenna moment of gearbox output shaft;

$\theta m 、 \theta \oiiint$ respectively are motor shaft angular position, angular velocity, angular acceleration;

$\theta a 、 \theta$ respectively are antenna shaft angular position, angular velocity, angular acceleration.

According to the bi-motor drive model, the motion differential equations are listed.

$$
\left\{\begin{array}{c}
u_{a}=e+R_{a} i_{a}+L_{a} \frac{d i_{a}}{d t} \\
e=K_{e} \theta_{m}^{\&} \\
2 M_{a}=2 K_{m} i_{a} \\
2 M_{a}=\frac{1}{N} 2 M_{l}+2 J_{m} \theta_{m}^{\alpha} \\
2 M_{l}=2 K_{g}\left(\frac{1}{N} \theta_{m}-\theta_{a}\right) \\
2 M_{l}=J_{a} \theta_{a}^{\alpha}
\end{array}\right.
$$

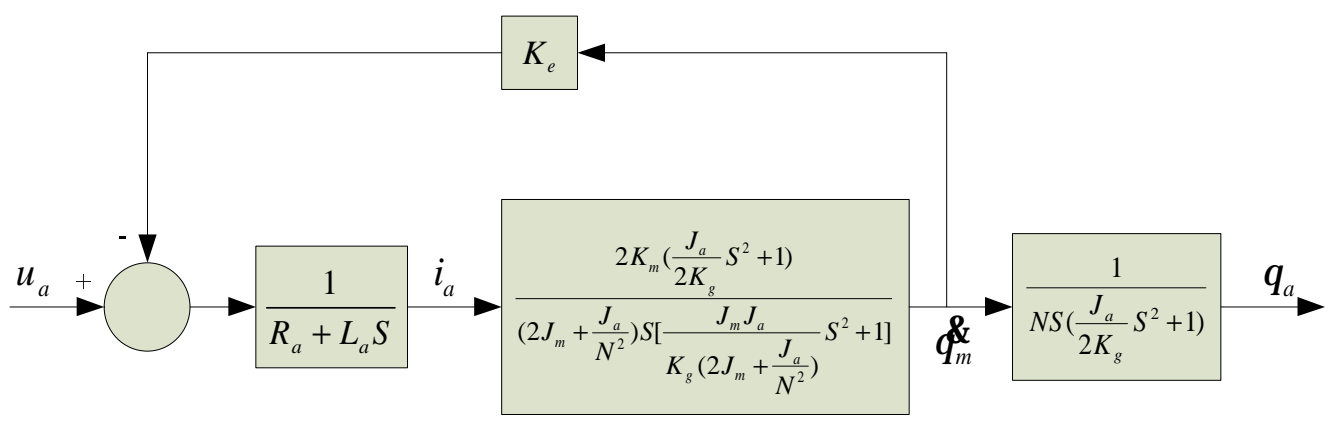

Fig. 2 Simplified block diagram of bi-motor control object

In the actual system, if the influence of back EMF is negligible, the transfer function of the control object in current loop is:

$$
\frac{I_{a}}{U_{a}}=\frac{1}{R_{a}+L_{a} S}
$$

The transfer function of the control object in speed loop, in addition to the current loop, and the transfer function from Ia to $\theta_{m}^{\alpha}$ is:

$$
W_{m}(S)=\frac{\theta_{m}^{\alpha}(S)}{I_{a}(S)}=\frac{2 K_{m}\left(\frac{J_{a}}{2 K_{g}} S^{2}+1\right)}{\left(2 J_{m}+\frac{J_{a}}{N^{2}}\right) S\left[\frac{J_{m} J_{a}}{K_{g}\left(2 J_{m}+\frac{J_{a}}{N^{2}}\right)} S^{2}+1\right]}
$$

Because the two motor working state is the same, this formula represents the transfer function from the armature current to the speed of each motor, the sum of rotational inertia of every motor itself and the sharing of the load inertia is:

$$
\begin{gathered}
J_{\Sigma}=J_{m}+\frac{J_{a}}{2 N^{2}} \\
J_{a}{ }^{\prime}=\frac{J_{a}}{2 N^{2}}
\end{gathered}
$$


In addition, by the expression of $W_{m}(S)$, due to the elastic load influence, in the numerator and the denominator each appeared an oscillation.

We suppose that:

$$
\omega_{L}=\sqrt{\frac{2 K_{g}}{J_{a}}}, \quad \omega_{f}=\sqrt{\frac{2 K_{g}\left(J_{m}+\frac{J_{a}}{2 N^{2}}\right)}{J_{m} J_{a}}}=\sqrt{1+\frac{J_{a}{ }^{\prime}}{J_{m}} \omega_{L}}
$$

$\omega_{L}$ is called locked rotor resonant frequency, $\omega_{f}$ is called the free rotor resonant frequency.

To obtain the transfer function of the control object is very difficult, sometimes even impossible. In the engineering design, mostly in the control object curve measured as the standard, the approximate equivalent to a low order system, the measured curve can be approximated by the following expressions are:

$$
G(S)=\frac{K_{v}\left(\frac{1}{\omega_{l}^{2}} S^{2}+1\right)}{S(T S+1)\left(\frac{1}{\omega_{f}^{2}} S^{2}+1\right)}
$$

\section{Modeling and analysis of current loop}

When the open loop gain of the current loop is high enough and the time constant is sufficiently small, the effects of DC motor back EMF feedback and back EMF compensation for current loop can be ignored.

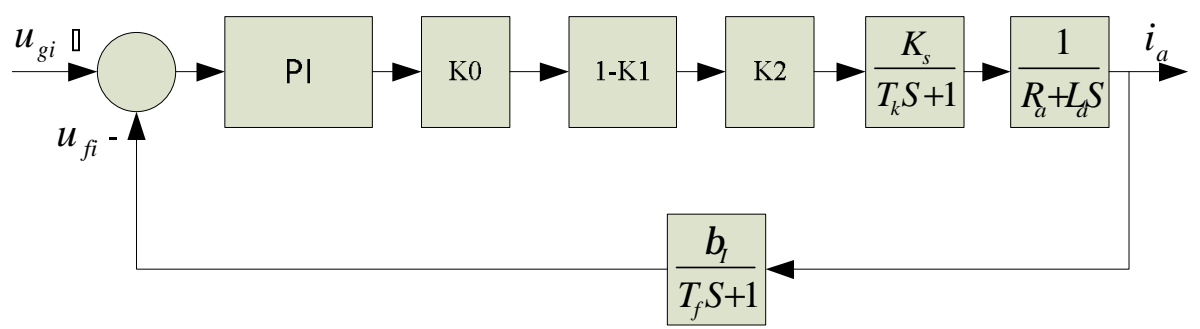

Fig. 3 The principle block diagram of current loop

K0: amplifier gain, in order to improve the response bandwidth of current loop in small input signal, K1: compensation coefficient of the back EMF; K2: gain of DC control signal amplitude limiting; Ks: voltage gain of silicon controlled rectifier; Tk: delay time constant of silicon controlled rectifier; Tf: filter time constant of current feedback branch; $\beta_{I}$ : current feedback coefficient;

Regulator is PI regulator, whose transfer function is:

$$
\frac{k(\tau S+1)}{\tau S}
$$

The transfer function of current loop:

$$
W(S)=\frac{K(\tau S+1)}{S\left(T_{e} S+1\right)\left(T_{\Sigma} S+1\right)}
$$

In the formula, $K=\frac{k K_{0}\left(1-K_{1}\right) K_{2} K_{s} \beta_{I}}{R_{a} \tau}, T_{\Sigma}=T_{k}+T_{f}, T e=L_{a} / R_{a}$.

\section{Modeling and analysis of speed loop}

For a bi-motor drive system, assuming the tow current loop are the same, the sum speed signal is feedback to the input of speed loop, amplified and regulated by PID regulation, the two speed loops can be integrated. 


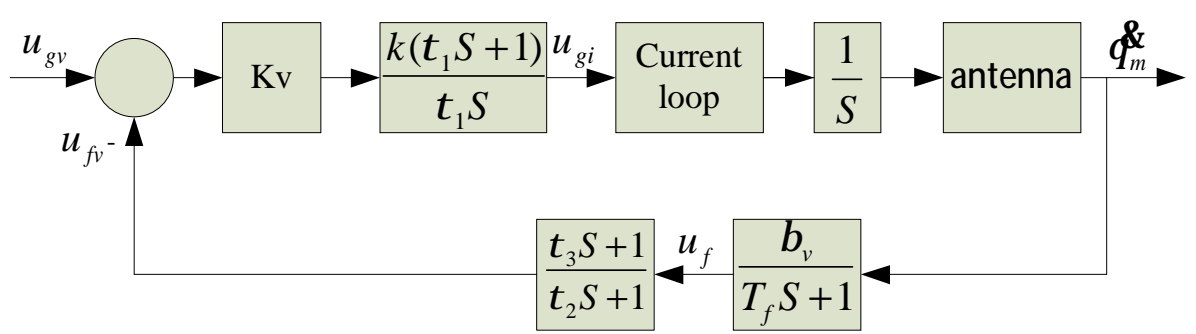

Fig. 4 The block diagram of speed loop

In the figure $4, \mathrm{Kv}$ is gain of speed loop; $\tau_{1} 、 \tau_{2} 、 \tau_{3}$ are parameters of PID regulator; $\mathrm{K}$ is gain of PID regulator; $\beta_{v}$ is speed feedback coefficient.

\section{Simulation of bi-motor servo drive system}

In this paper, by computer aided design and simulation function of Matlab/Simulink, bi-motor servo drive system of a radar range branch is simulated and tested.

\section{Simulation of current loop}

For a radar, we select the appropriate parameters, the simulation structure of current loop as shown in Figure 5 is established by using Simulink.

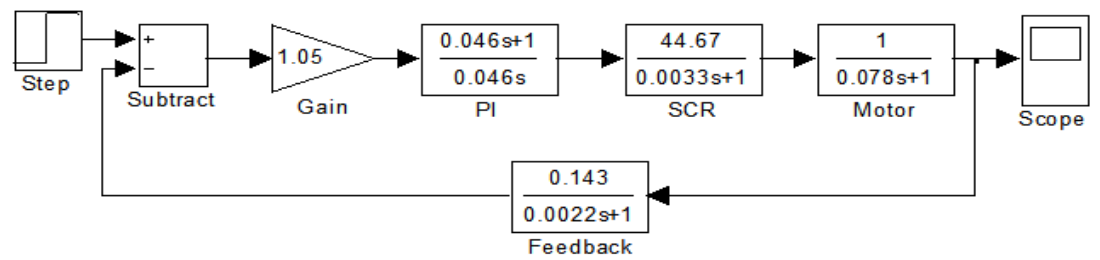

Fig. 5 The simulation structure of current loop

Through Matlab simulation, the step response results are that the rise time is about $15 \mathrm{~ms}$, the overshoot is $15 \%$, and the number of oscillations is 1 , the bandwidth is $25 \mathrm{~Hz}$. It will not arouse to large lag for the speed loop, in line with the actual situation of the system.

\section{Simulation of speed loop}

In the radar servo system with large inertia antenna, the influence to dynamic performance by structural resonance frequency must be considered. Figure $7 \mathrm{a}$ is potter map of a type of radar antenna.

The structure of resonant frequency, namely fixed rotor resonant frequency is about $4.25 \mathrm{~Hz}$ $(26.7 \mathrm{rad} / \mathrm{s})$, free rotor resonant frequency is about $9.87 \mathrm{~Hz}(62.0 \mathrm{rad} / \mathrm{s})$.

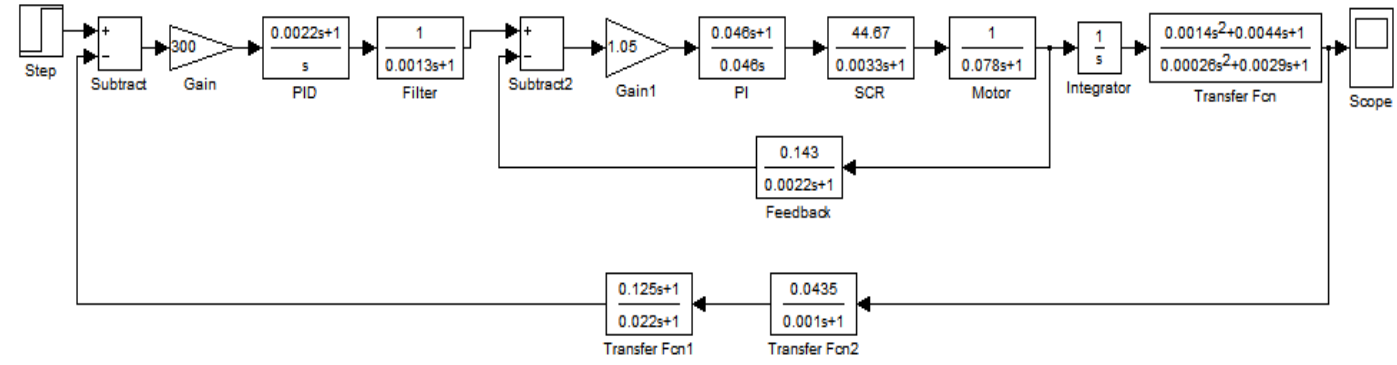

Fig. 6 The simulation structure of speed loop 


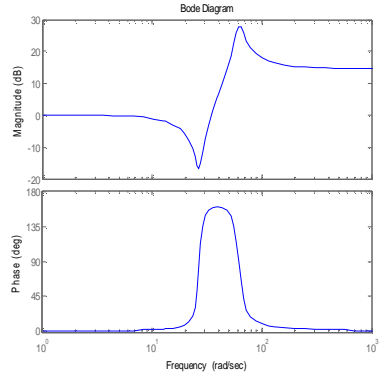

a

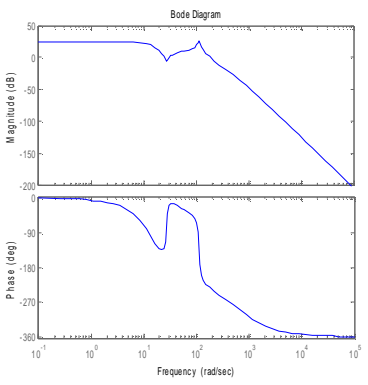

b

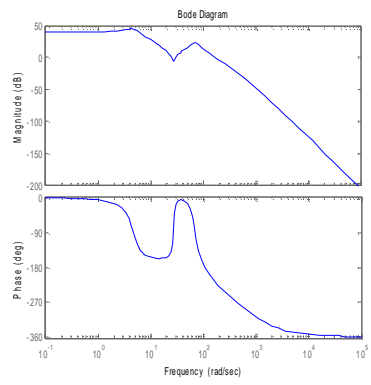

$\mathrm{C}$

Fig. 7 The bode figure of system

Figure $7 \mathrm{~b}$ is Potter map of speed closed loop. It can be seen from the graph, as long as the speed loop bandwidth is selected appropriate, and whose bandwidth is not beyond the range of antenna structure resonance frequency, the system is stable.

The transient response characteristics of the speed loop is shown in Figure 8a, it can be seen from the figure, the rise time is about $200 \mathrm{~ms}$, the overshoot is $10 \%-15 \%$, the number of oscillations is 1 , the bandwidth is about $2.25 \mathrm{~Hz}$, they meet the index of the system requirements.

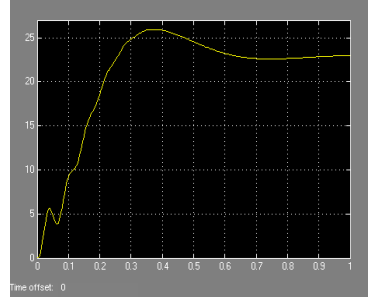

a

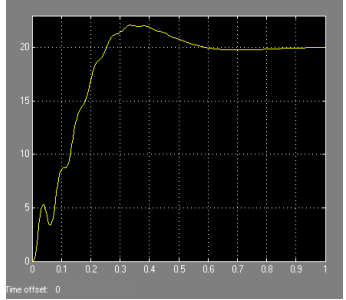

b

Fig. 8 The step response of speed loop

In the speed loop, if the antenna structure changes, the resonant frequency of the antenna will reduce, or if the performance of the motor for speed feedback reduces, it will cause Potter diagram curve of the system to deform in advance. If the deformation falls within the bandwidth range of the speed loop, it will cause the system instability, thereby cause the antenna oscillation.

If the feedback coefficient of the tachometer of a radar decreases caused by the aging, it will make the amplitude frequency characteristics and phase frequency characteristics of the speed loop distortion, lead to transient response performance degradation, and lead to angle tracking of antenna oscillation, seriously lead to the system unstable. The potter chart is shown in figure 7c.

Figure $8 \mathrm{~b}$ is the step response curve of the speed loop when the tachometer feedback coefficient has decreased. It can be seen from the chart, when speed feedback coefficient changes, compared with the normal conditions, rise time and overshoot of the step response curve change a little, the bandwidth of the speed loop changes a little, but there is oscillation trend as the antenna rotation. So the change of speed feedback coefficient must be controlled in a certain range, if it is beyond this range, it will cause the antenna oscillation.

\section{Conclusion}

In this paper, the author makes the model and analyses of the bi-motor servo drive system, and carries out a simulation study separately to the current loop and speed loop. Simulation results show that if the load characteristics and feedback components performance decreases, it will directly affect the transient response characteristics of servo drive system. Thus we can judge and make the measurements of improving system performance by the analysis of the potter chart and test of transient response characteristics. 


\section{References}

[1] Li Liansheng, The radar servo system[M], Beijing, National Defense Industry Press, 1983.

[2] Wang Dechun, Ding Jiahui, Chen Wangdong, Precision tracking radar technology[M], Beijing, Publishing house of electronics industry, 2006.

[3] Xue Dingyu, Chen Yangquan, Technology and application, system simulation based on Matlab/Simulink [M], Beijing, Tsinghua University press, 2002.

[4]Li Qiong, Wang Shaoping, Liang Lei, The Structure, Modeling and Simulation of Optical Drive Servo System[J], Piezoelectectrics and Acoustooptics, 2007, 213-218.

[5]Luo Xiao, Yang Wusen, Dynamic Modeling and Nonlinear Simulation Analysis of Gear Backlash of Dual-motor Drive Servo System[J],Electronic information and Automatic control, 2013, 106-109.

[6]Fan Weihua, Zhao Guofeng, Chen Qingwei , Hu Weili, Design of neural network controller of dual-motors driving Servo system[J], Electric machines and Control, 2006, 261-268.

[7]Hu Mailing , Zhang Guanghui , Wei Ping , Liu Xingsong, Modeling and Analysis of Double-Motor Driven Servo System[J], Journal of gun launch and Control, 2008, 93-96. 